\title{
Glomerular angiotensinogen protein is enhanced in pediatric IgA nephropathy
}

\author{
Masanori Takamatsu • Maki Urushihara • \\ Shuji Kondo • Maki Shimizu • Tetsuo Morioka • \\ Takashi Oite • Hiroyuki Kobori • Shoji Kagami
}

Received: 14 November 2007 / Revised: 30 January 2008 / Accepted: 18 February 2008 / Published online: 18 April 2008

(C) The Author(s) 2008

\begin{abstract}
Enhanced intrarenal renin-angiotensin system (RAS) is implicated in the development and progression of renal injury. To investigate whether angiotensinogen (AGT) expression is involved in glomerular RAS activity and glomerular injury, we examined glomerular AGT expression and its correlation with expression of other RAS components, and levels of glomerular injury in samples from patients with immunoglobulin A nephropathy (IgAN) (23) and minor glomerular abnormalities (MGA) (8). Immunohistochemistry showed that AGT protein was highly expressed by glomerular endothelial cells (GEC) and mesangial cells in nephritic glomeruli of IgAN compared with glomeruli of MGA. Levels of glomerular AGT protein were well correlated with levels of glomerular angiotensin II (ang II), transforming growth factor- $\beta$ (TGF- $\beta$ ), $\alpha$-smooth-muscle actin, glomerular cell
\end{abstract}

M. Takamatsu $\cdot$ M. Urushihara $\cdot$ S. Kondo $\cdot$ M. Shimizu $\cdot$

S. Kagami $(\bowtie)$

Department of Pediatrics, The Institute of Health Bioscience,

The University of Tokushima Graduate School,

Kuramoto-cho-3-chome,

Tokushima 770-8503, Japan

e-mail: kagami@clin.med.tokushima-u.ac.jp

T. Morioka $\cdot$ T. Oite

Department of Cellular Physiology, Institute of Nephrology,

Niigata University Graduate School of Medical

and Dental Sciences,

Niigata, Japan

H. Kobori

Department of Medicine,

Tulane University Health Sciences Center,

New Orleans, LA, USA

\section{H. Kobori}

Department of Physiology,

Tulane University Health Sciences Center,

New Orleans, LA, USA number, and glomerulosclerosis score but not with those of glomerular angiotensin-converting enzyme and ang II type 1 receptor. Real-time polymerase chain reaction (RT-PCR) and Western blot analyses using cultured human GEC indicated that ang II upregulated AGT messenger ribonucleic acid (mRNA) and protein expression in a dose- and timedependent manner. These data suggest that activated glomerular AGT expression is likely involved in elevated local ang II production and, thereby, may contribute to increased TGF- $\beta$ production and development of glomerular injury in IgAN. Augmentation of GEC-AGT production with ang II stimulation might drive further glomerular injury in a positive-feedback loop.

Keywords Renin-angiotensin system .

Transforming growth factor- $\beta$.

Glomerular endothelial cells $\cdot \alpha$-smooth muscle actin .

Mesangial cells

\section{Introduction}

Clinical and experimental studies have provided considerable evidence for the role of the renin-angiotensin system (RAS) in the development of hypertension and progression of renal diseases $[1,2]$. It is now established that all components of the RAS are widely distributed in several tissues, including the kidney [3, 4]. The local RAS in the kidney has several pathophysiologic functions for not only regulating blood pressure but also renal cell growth and production of glomerulosclerosis, which is involved in the development of renal fibrosis [5-8]. Indeed, previous studies have shown beneficial effects of angiotensin-converting enzyme inhibitor (ACEi) and/or angiotensin II (ang II) type 1 receptor (AT1R) blocker (ARB) on various renal diseases in rats and humans, 
often to an extent far greater than their effect on blood pressure [9-11].

IgA nephropathy (IgAN) is now recognized to be the most common form of glomerulonephritis worldwide. It has been reported that more than $30 \%$ of cases progress to end-stage renal failure within 20 years $[12,13]$. In pediatric IgAN, glomerular lesion is characterized by an increased number of mesangial cells (MC) and accumulation of mesangial extracellular matrix (ECM), and this pathological change is thought to be an early and active lesion for IgAN [14]. It is reported that treatment with ACEi and/or ARB provides antiproteinuric and renoprotective effects in normotensive pediatric patients with IgAN, suggesting that intrarenal action of ang II may play a critical role in the progression of pediatric IgAN $[15,16]$. Recently, a real-time polymerase chain reaction (RT-PCR) study using isolated glomeruli with IgAN revealed an increased expression of RAS components such as renin, angiotensinogen (AGT), and ACE, possibly followed by the production of ang II, an effector molecule of RAS, suggesting that the glomerular RAS activation might occur under this disease setting [17]. In this study, AGT messenger ribonucleic acid (mRNA) levels were positively correlated with the mRNA expression of renin, ACE, and a fibrogenic cytokine transforming growth factor- $\beta$ (TGF- $\beta$ ). AGT is the sole substrate for the RAS pathway, and it has been reported that AGT is present within the kidney and ang II is produced locally, independent of the circulating RAS [18]. Interestingly, the increased expression of intrarenal AGT is directly involved in the disease progression in several rat models of renal injury [19-22]. However, little information is available about protein expression in an RAS cascade from AGT to ang II and the association between glomerular RAS activation and pathological changes in childhood IgAN.

Therefore, in this study, the glomerular protein expression of RAS components such as AGT, ACE, ang II, and AT1R, as well as their association with the index of glomerular injury were examined to clarify the role of the RAS cascade, especially that of AGT in glomerular lesions of pediatric IgAN. Subsequently, we investigated the amplification mechanism of glomerular AGT expression leading to an increase of glomerular RAS activity using cultured human glomerular endothelial cells (GEC).

\section{Materials and methods}

\section{Kidney tissue}

Renal tissues used for this study were obtained from 23 children with IgAN and eight with minor glomerular abnormalities (MGA) by biopsy. Diagnosis of renal diseases was made based on studies of tissue by light, electron, and immunofluorescence microscopy according to the classification of the World Health Organization (WHO) [23]. This classification defined diffuse mesangial proliferation as $>80 \%$ of glomeruli showing moderate or severe mesangial cell proliferation, i.e. more than three cells per peripheral mesangial area, and focal mesangial proliferation as up to $80 \%$ of glomeruli showing the same changes described above. IgAN was diagnosed as a mesangial proliferative glomerulonephritis with predominant IgA deposit in the mesangium. Patients diagnosed with IgAN showed diffuse mesangial proliferation (15) or focal mesangial proliferation (8). Patients diagnosed with MGA showed normal glomerular morphology and negative immunofluorescence but had mild proteinuria or microscopic hematuria. All subjects with other disorders, including Henoch-Schönlein purpura, systemic lupus erythematosus, and chronic liver disease, were excluded. The control tissue (5) consisted of normal cortex taken from kidneys resected for localized neoplasms. Blood pressure, serum creatinine, and creatinine clearance were normal in all participants at the time of biopsy. Patients had not been previously treated with either ACEi, ARB, glucocorticosteroids, or immunosuppressive agents. The study protocol was approved by the ethics committee of the University of Tokushima Graduate School, and written informed consent was obtained from all patients and/or their parents in all cases. The clinical laboratory parameters, including age, term from onset of urinary abnormalities to renal biopsy, blood pressure, proteinuria, hematuria index, serum creatinine, and 24-h creatinine clearance were determined at the time of biopsy. Based on examination of urine samples, the severity of hematuria was classified as follows: $0,0-5$ erythrocytes/high-power field (HPF); 1+, 5-30 erythrocytes/HPF; 2+, >30 erythrocytes/ HPF; $3+$, macroscopic hematuria, as previously described [24]. Clinical data are presented in Table 1.

Histological scoring of glomerular cell proliferation, glomerulosclerosis, and tubulointerstitial changes

For light-microscopic examination, the biopsied tissues were fixed in $10 \%$ buffered formalin and embedded in paraffin. Paraffin sections $(3 \mu \mathrm{m})$ were stained with periodic acidSchiff reagent. All glomeruli in each section (usually 10-26) were coded and read by a blinded observer. The number of cells in at least four equatorially cut glomeruli in each section was counted, and the average number of cells was used as an indicator of glomerular cell proliferation, as previously described [25]. The glomerulosclerosis (GS) score was determined using a previously described method with a modification [26]. The percentage of each glomerulus occupied by mesangial ECM was estimated and assigned a code as follows: 0 , absent; $1+, 1-5 \% ; 2+, 5-25 \% ; 3+, 25-50 \%$; 4+, $>50 \%$; and the mean value per specimen was calculated [27]. 
Table 1 Clinical data of patients

$M G A$ minor glomerular abnormalities, IgAN immunoglobulin A nephropathy

Data are means \pm standard deviation

$* P<0.01$ vs MGA

\begin{tabular}{lcc}
\hline & MGA & IgAN \\
\hline Number & 8 & 23 \\
Age (years) & $13.1 \pm 1.9$ & $13.0 \pm 3.1$ \\
Term from onset of urinary abnormalities to & $1.5 \pm 1.2$ & $0.9 \pm 0.7$ \\
$\quad$ renal biopsy (years) & & \\
Systolic/diastolic blood pressure (mmHg) & $112.1 \pm 9.5 / 62.6 \pm 7.5$ & $111.0 \pm 10.4 / 61.3 \pm 7.9$ \\
Proteinuria (g/day) & $0.07 \pm 0.04$ & $0.37 \pm 0.26^{*}$ \\
Hematuria index & $0.93 \pm 0.37$ & $1.89 \pm 0.70^{*}$ \\
Serum creatine (mg/dl) & $0.54 \pm 0.14$ & $0.54 \pm 0.15$ \\
24-h creatine clearance (ml/min per $\left.1.73 \mathrm{~m}^{2}\right)$ & $100.5 \pm 23.5$ & $103.8 \pm 29.9$ \\
\end{tabular}

To evaluate the level of tubulointerstitial change, three to five fields of the cortical interstitium in each section were examined, as previously described [28]. In each field, tubulointerstitial change, like tubular atrophy and interstitial broadening, were graded as follows: 0, representing involvement of $<5 \%$; $1+$, involvement between 5 and $20 \%$; $2+$, involvement between 20 and $40 \%$; $3+$, involvement of $>40 \%$.

\section{Antibodies}

Chicken IgY-rich fraction against highly purified full-length human plasma AGT (anti-AGT antibody) was used for immunohistochemistry [29]. Mouse monoclonal anti-ACE antibody, rabbit polyclonal anti-ang II serum, and rabbit polyclonal anti-AT1R antibody were purchased from Chemicon International (Temecula, CA, USA), IgG corporation (Nashville, TN, USA) and Santa Cruz Biotechnology (Santa Cruz, CA, USA), respectively. Mouse monoclonal anti- $\alpha$-smooth-muscle ( $\alpha$-SM) actin antibody (1A4) was purchased from Sigma-Aldrich (St. Louis, MO, USA) and was used to detect activated human MC. Rabbit polyclonal anti-TGF- $\beta$ antibody was a kind gift from Dr. Kawachi (Niigata University, Niigata, Japan).

\section{Immunohistochemistry}

The glomerular expression of AGT or AT1R was detected by an immunoperoxidase technique, as previously described [27]. Briefly, the sections $(3 \mu \mathrm{m})$ were incubated with the primary antibodies overnight at $4^{\circ} \mathrm{C}$. After rinsing, the sections were incubated with either biotinylated anti-chicken IgG (for AGT) (Vector Labs, Burlingame, CA, USA) or biotinylated anti-rabbit IgG (for AT1R) (Vector Labs). After rinsing, the sections were incubated with avidin-biotinperoxidase complex (ABC Elite; Vector Labs), followed by 3,3'-diaminobenzidine (Dojindo, Kumamoto, Japan). Each section was counterstained with Mayer's hematoxylin (Wako, Tokyo, Japan), dehydrated, and coverslipped.

In double-staining experiments, 3- $\mu \mathrm{m}$ frozen sections stained with chicken anti-AGT antibody, and fluorescein- isothiocyanate (FITC)-labeled donkey anti-chicken IgG antibody (Jackson ImmunoResearch Laboratories, West Grove, PA, USA) were further incubated with either mouse anti-CD31 antibody (DAKO, Glostrup, Denmark), mouse monoclonal anti- $\alpha$-SM actin antibody (1A4), or mouse monoclonal synaptopodin antibody (G1D4; Progen Biotechnik, Heidelberg, Germany), followed by an appropriate tetramethylrhodamine-isothiocyanate (TRITC)-coupled donkey anti-mouse antibody (Jackson Immuno-Research Laboratories). Indirect immunofluorescent staining was performed using the following primary antibodies: anti-ACE antibody, anti-ang II antiserum, anti- $\alpha$-SM actin antibody, and antiTGF- $\beta$ antibody. FITC-labeled donkey anti-rabbit IgG antibody, donkey anti-mouse IgG, and TRITC-labeled donkey anti-mouse IgG (Jackson ImmunoResearch Laboratories) were used as the secondary antibodies. Frozen sections were incubated with primary antibodies for $1 \mathrm{~h}$, and then secondary antibodies, appropriately. To evaluate the level of glomerular staining with each antibody, we performed semiquantitative analysis. The immunostaining of AGT was graded as follows: 0 , very weak or absent capillary or mesangial staining; 1+, weak capillary staining with $1-25 \%$ focally increased capillary staining; 2+, 25-50\% of glomerular tuft demonstrating strong capillary and mesangial staining; 3+, 50-75\% of glomerular tuft demonstrating strong capillary and mesangial staining; $4+, 75 \%$ or more of the glomerular tuft demonstrated strong capillary and mesangial staining, as previously described [25]. The immunostaining of ACE, ang II, AT1R, TGF- $\beta$, and $\alpha$-SM actin was graded as follows: 0 , no staining; $1+$, weak staining; $2+$, mild staining; $3+$, moderate staining; 4+, marked staining. At least four equatorially sectioned glomeruli in each section were evaluated blindly by two separate investigators. The individual immunostaining score was determined by calculating the average value per single glomerulus [27].

Cell culture

Cultured human GEC derived from normal human kidneys were purchased from Cell System (Kirkland, WA, USA). 
Human GEC were cultured on culture flask coated with type 1 collagen, as described previously [30]. Human GEC were used at the sixth to eighth passage in our experiments. At about $70-80 \%$ confluency, GEC growth was arrested by incubation for $48 \mathrm{~h}$ in serum-free medium and then treated with ang II at increasing concentrations $\left(0,10^{-11}, 10^{-9}\right.$, $10^{-7} \mathrm{M}$ ) for 3-12 $\mathrm{h}$ to detect mRNA or protein, respectively. In addition, a time-course study was undertaken to assess changes in AGT mRNA expression at $0,1,3,6,12$, and $24 \mathrm{~h}$ and protein expression at $0,6,12$, and $24 \mathrm{~h}$ with $10^{-7} \mathrm{M}$ ang II-treated GEC. Cells were harvested for RNA extraction and total cell extract preparation.

Western blot

Human GEC cultures were dissolved in radioimmunoprecipitation assay (RIPA) buffer. Protein samples $(25 \mu \mathrm{g})$ were separated by $12.5 \%$ sodium dodecyl sulfate polyacrylamide gel electrophoresis (SDS-PAGE) and transferred onto nitrocellulose membranes (Amersham Bioscience, Piscataway, NJ, USA). The membranes were probed with anti-AGT antibody $(1: 1,000)$ and then incubated with a horseradish peroxidase-conjugated rabbit anti-chicken/ turkey IgG (H+L) (1:2,000; Zymed Laboratories, San Francisco, CA, USA). Equivalent protein loading for each lane was confirmed by stripping and reblotting each membrane for $\beta$-actin $(1: 10,000$; Sigma-Aldrich). Immunoreactive proteins were detected with an enhanced chemiluminescence detection system (Amersham Corp., Arlington Heights, IL, USA). The antibodies were tested on human purified AGT (Calbiochem, Merck, Darmstadt, Germany) as control, where two bands between 50 and $60 \mathrm{kD}$ were detected [31]. Bands were quantitated by ImageJ 1.33u (National Institute of Health), and the fold expression was indicated as the relative protein level.

\section{RT-PCR}

Total RNA (500 ng) extracted from human cell cultures was reverse transcripted using the SYBR ExScript RT-PCR Kit (TaKaRa, Kyoto, Japan). The complementary DNAs (cDNAs) were amplified by RT-PCR with primers for human AGT (forward 5'-TCTCCCCGGACCATCCA-3' and reverse $5^{\prime}$-TGCTCAATTTTTGCAGGTTCAG-3') and glyceraldehyde-3 phosphate dehydrogenase (G3PDH) (forward 5'-GAAGGTGAAGGTCGGAGT-3' and reverse 5'-TGGCAACAATATCCACTTTACCA-3') as an internal control, using iCycler Thermal Cycler (BioRad, Hercules, CA, USA) [17, 32]. Melting curve analysis and PAGE confirmed the specificity of the amplification products. Semiquantification data were analyzed with iCycler analysis software and expressed as the ratio between starting the quantity mean of the target and the G3PDH gene.
Statistical analysis

Results are presented as means \pm standard deviation (SD). StatView (SAS institute) was used for statistical analyses. Between-group differences were assessed by the unpaired $t$ test. The correlation of two variations was evaluated using Pearson's or Spearman's correlation coefficients. Western blot and RT-PCR analyses were performed at least three times, and representative results are shown in the figures. The mRNA expression or protein synthesis of AGT in cultured cells was analyzed by $t$ test. Values of $P<0.05$ were considered statistically significant.

\section{Results}

Clinical data and histologic changes in patients

Clinical data in patients with MGA or IgAN are listed in Table 1. There were no significant differences between MGA and IgAN patients for age, follow-up term from onset of urinary abnormalities, blood pressure, serum creatinine, and creatinine clearance at the time of biopsy. Mean urine protein excretion was significantly higher in IgAN patients than that in MGA patients $(P<0.01)$. The extent of glomerular cellularity, ECM accumulation, and tubulointerstitial changes were semiquantified in both patient groups and are shown in Fig. 1. The glomerular cell number and GS score were significantly higher in IgAN patients compared with those in MGA patients (MGA vs IgAN; mean cell number: 66.1 vs $77.9, P<0.05$; mean GS score: 0.73 vs $1.58, P<0.01)$. Tubular atrophy and interstitial broadening were faintly present in IgAN patients but not in MGA patients. However, there was no significant difference in tubulointerstitial score change between the two groups (mean score: 0.00 in MGA vs 0.26 in $\operatorname{IgAN}, P=0.188$ ).
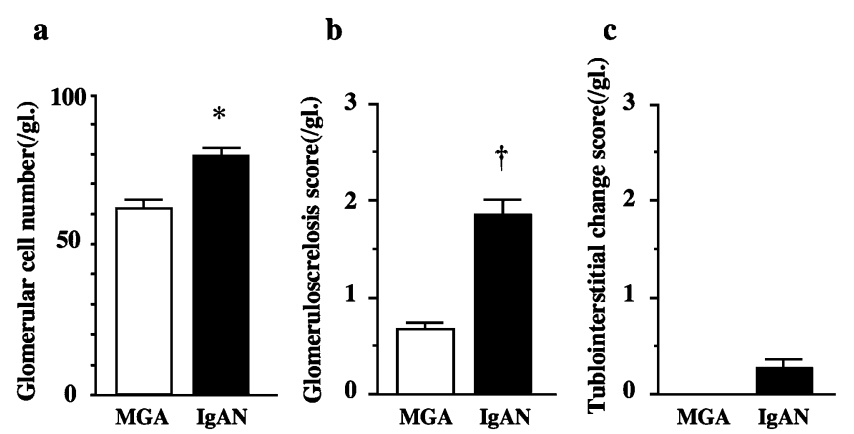

Fig. 1 The scorings of glomerular cell number (a), glomerulosclerosis (b), and tubulointerstitial changes (c) in the biopsies. Glomerular cell count and glomerulosclerotic indexes except tubulointerstitial changes significantly increased in immunoglobulin A nephropathy (IgAN) (a)

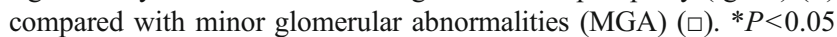
vs MGA; $\dagger P<0.01$ vs MGA 
Expression of RAS components, TGF- $\beta$, and $\alpha$-SM actin in patients with MGA or IgAN

The glomerular localization of RAS components was studied by immunohistochemistry in renal biopsies from patients with either IgAN or MGA. Immunostaining of AGT was detected mainly in proximal tubules and weakly in GEC lining capillary walls in MGA specimens, as in normal tissues (Fig. 2a). This renal localization of AGT was consistent with rat renal localization of AGT, as previously described [33]. Of note, the staining level of AGT was enhanced in a typical endocapillary pattern, including changes of GEC and MC in IgAN patients (Fig. 2b). No positive immunostaining of AGT was seen when a chicken antibody against AGT, absorbed by preincubation with excess human purified AGT, was used as the primary antibody or when the primary antibody was omitted (Fig. 2c). The double immunostaining in IgAN showed that AGT was primarily detected on GEC, to a lesser extent on $\mathrm{MC}$, and only faintly on podocytes, supporting the glomerular localization of AGT in an immunoperoxidase technique (Fig. 2d-1).

ACE and ang II expression were examined by immunofluorescence, as specific staining was not detected by the immunoperoxidase technique. Faint immunofluorescence of ACE and ang II was observed in glomeruli from patients with MGA, as in normal tissues (Fig. 3a,c). In IgAN patients, staining for ACE tended to increase in an endocapillary pattern, but that of ang II was enhanced in GEC and MC of nephritic glomeruli (Fig. 3b,d). A similar staining level of AT1R was found in glomeruli, particularly $\mathrm{MC}$, in specimens from patients with MGA or IgAN, and in control kidney tissues (Fig. 3e,f).

A semiquantitative analysis of glomerular staining for AGT, ACE, ang II, and AT1R revealed that the level of glomerular AGT (mean score 1.89) and ang II (mean score

Fig. 2 Glomerular expression of angiotensinogen (AGT) and its localization in the biopsies. Kidney sections were immunostained with anti-AGT antibody in patients with minor glomerular abnormalities (MGA) (a) or immunoglobulin A nephropathy $(\operatorname{IgAN})$

(b) (original magnification $x$ 200). Positive immunostaining of AGT could not be detected when a chicken antibody against AGT, absorbed by preincubation with excess human purified AGT, was used as the primary antibody (c). To identify glomerular localization of AGT, double immunofluorescence staining of AGT (green; $\mathbf{d}, \mathbf{g}, \mathbf{j}$ ) with either glomerular endothelial cells (GEC) marker CD31 (red; e), activated mesangial cells (MC) $(\mathrm{red} ; \mathbf{h})$, or podocyte marker $($ red; $\mathbf{k})$ in IgAN was performed. Merged images show that AGT clearly colocalized on GEC (f), weakly on activated MC (i), and only faintly on podocyte (I) in IgAN $(\times 400)$
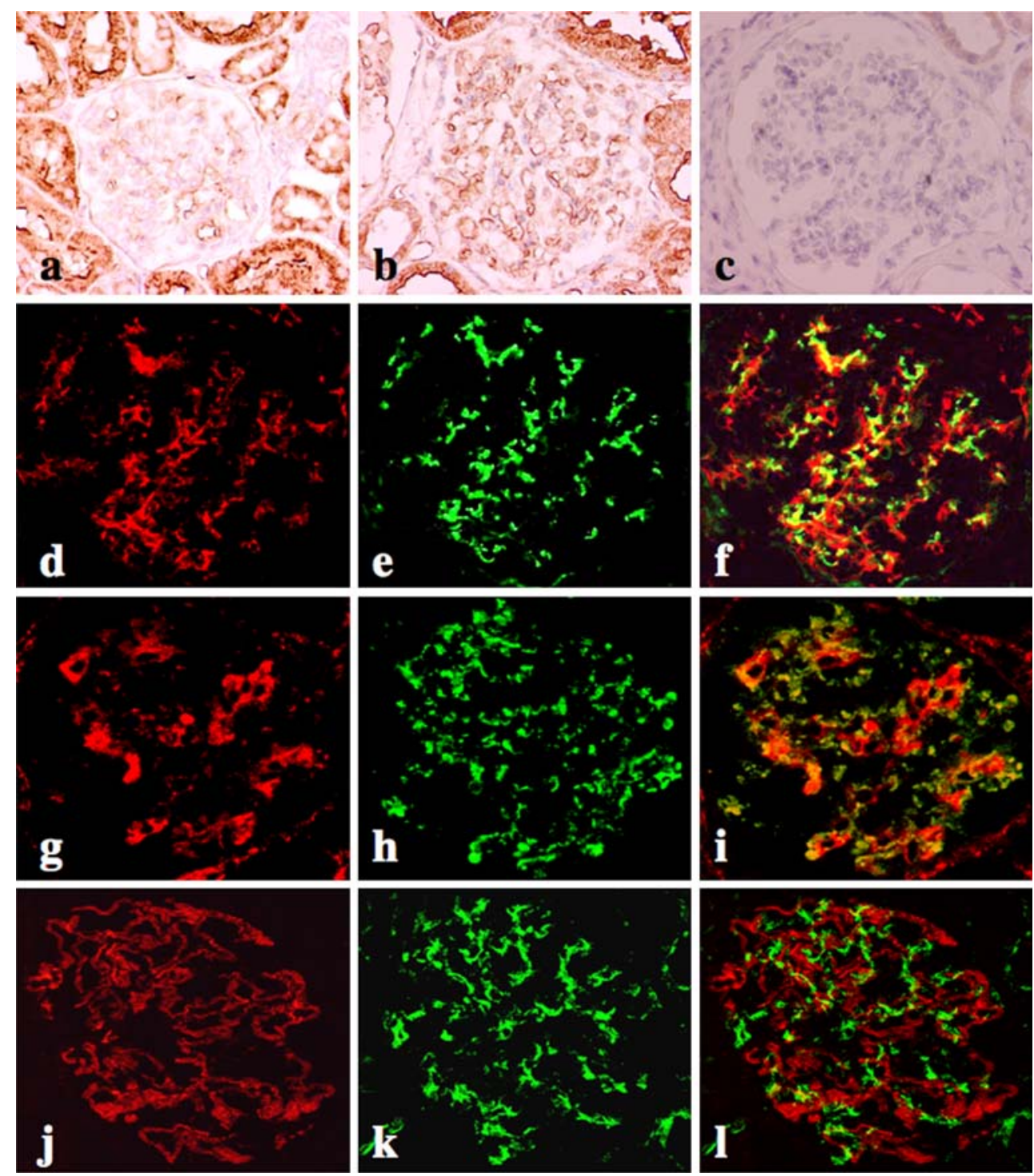

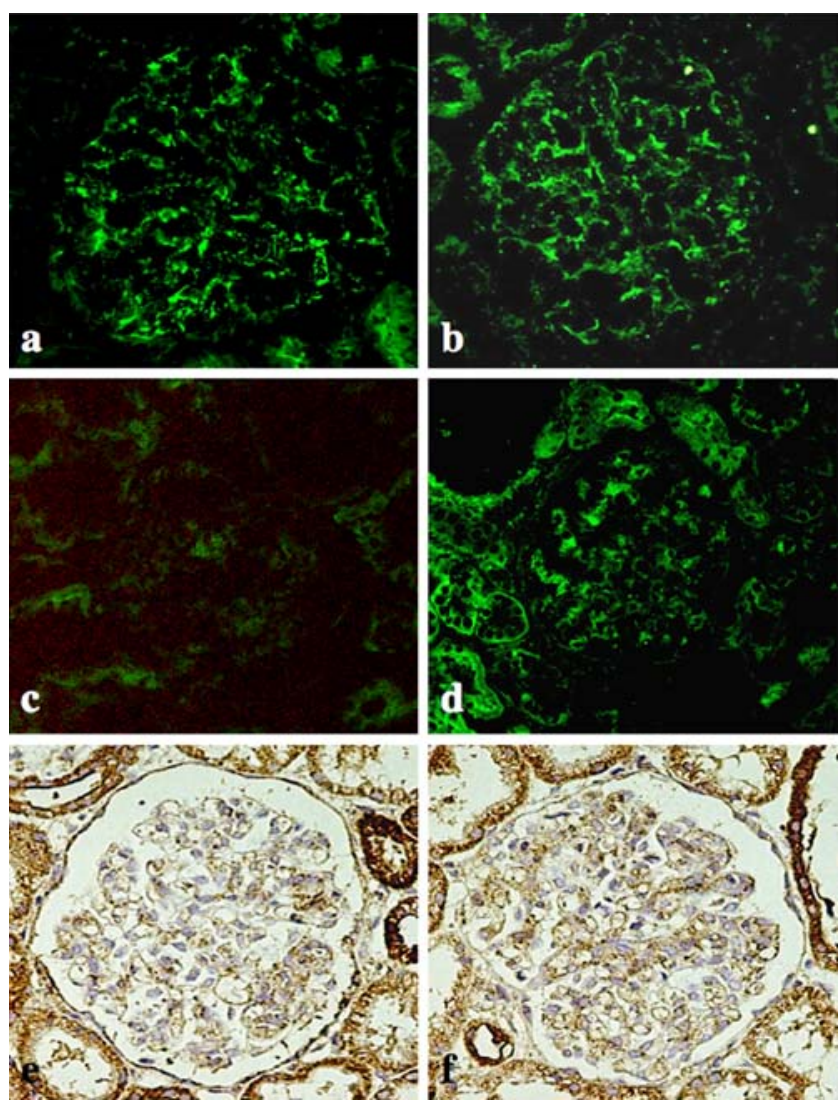

Fig. 3 Glomerular expression of components of the renin-angiotensin system (RAS) in the biopsies. Kidney sections were immunostained with either anti-angiotensin-converting enzyme antibody (a, b), antiangiotensin II antibody (c, d) or anti-angiotensin II type 1 receptor antibody (e, f) in patients with minor glomerular abnormalities (MGA) (a, c, e) or immunoglobulin A nephropathy $(\operatorname{IgAN})(\mathbf{b}, \mathbf{d}, \mathbf{f})$ (original magnification, $\times 200$ )

2.09) but not ACE (mean score 0.96) was significantly enhanced in the IgAN group compared with the MGA group (mean scores for AGT, ang II, ACE 0.93, 1.00, 0.50, respectively) $(P<0.01)$ (Fig. 4). The expression level of AT1R tended to be lower, but not statistically significant, in

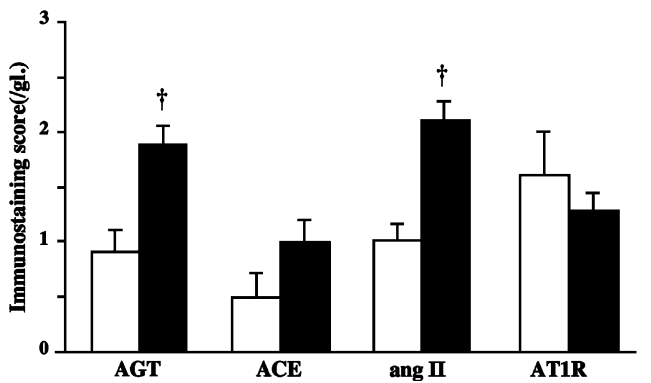

Fig. 4 Semiquantitative assessment of immunostaining of angiotensinogen (AGT), angiotensin-converting enzyme (ACE), angiotensin II (ang II) and ang II type 1 receptor (AT1R) in the kidney sections from patients with minor glomerular abnormalities (MGA) ( $\square$ ) or immunoglobulin A nephropathy $(\operatorname{IgAN})(\square)$. Data are means \pm standard deviation. $\dagger P<0.01$ vs MGA patients with IgAN compared with the MGA group (mean score 1.63 in MGA vs 1.26 in IgAN).

The expression of TGF- $\beta$ and $\alpha$-SM actin, potential factors associated with GN progression, was greatly enhanced in the expanded mesangial regions in patients with IgAN in contrast with patients with MGA (Fig. 5a-d). Semiquantitative analysis indicated that the levels of TGF$\beta$ and $\alpha$-SM actin expression were significantly higher in the IgAN group than in the MGA group (mean score 0.50 and 0.63 in MGA vs 1.91 and 1.91 in $\operatorname{IgAN} ; P<0.01)$ (Fig. 5e).

Correlation of RAS components with pathologic changes in patients with MGA or IgAN

Possible relationships between glomerular RAS activation and injury in diseased kidney were investigated to examine the correlations between the expression of RAS components, ang II-inducible factors, TGF- $\beta$ and $\alpha$-SM actin histologic
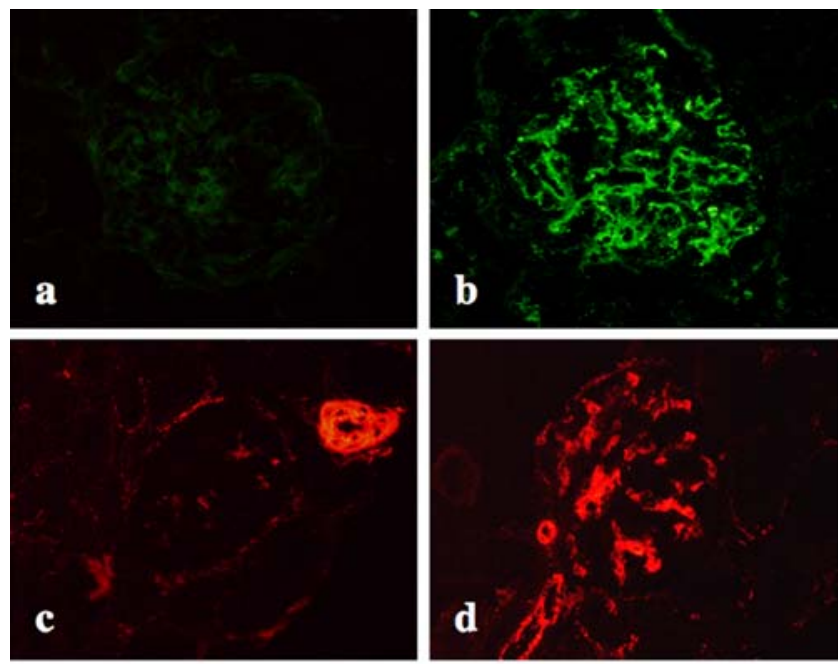

e

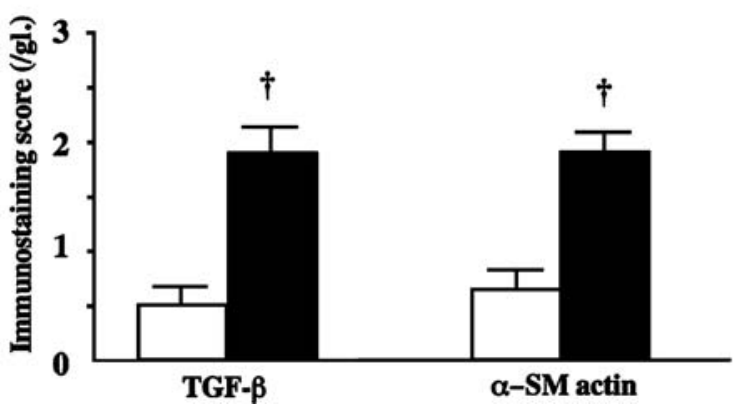

Fig. 5 Glomerular expression of transforming growth factor- $\beta$ (TGF$\beta)$ and $\alpha$-smooth-muscle $(\alpha$-SM) actin in biopsies. Kidney sections were immunostained with either anti-TGF- $\beta$ antibody (a, b) or $\alpha$-SM actin (c, d) in patients with minor glomerular abnormalities (MGA) (a, c) or immunoglobulin A nephropathy $(\operatorname{IgAN})(\mathbf{b}, \mathbf{d})$ (original magnification $\times 200$ ). Semiquantitative assessment of immunofluorescence staining of TGF- $\beta$ and $\alpha$-SM actin immunostaining in patients with MGA ( $\square$ ) or IgAN ( $\mathbf{\square}) . \dagger P<0.01$ vs MGA 
findings, and urinary findings (proteinuria and hematuria) in patients with MGA or IgAN (Fig. 6). The glomerular AGT expression was positively correlated with ang II $(r=+0.711$, $P<0.001)$ (Fig. 6a), TGF- $\beta(r=+0.723, P<0.001)$ (Fig. 6b), $\alpha$-SM actin $(r=+0.565, P<0.005)$ (Fig. 6c), glomerular cell number $(r=+0.542, P<0.005)$ (Fig. 6d), GS score $(r=+0.768, P<0.001)$ (Fig. 6e), and proteinuria $(r=+0.436$, $P<0.05$ ) (Fig. 6f). Similarly, glomerular ang II level showed a significant positive correlation with that of TGF- $\beta \quad(r=+0.782, \quad P<0.001) \quad($ Fig. $6 \mathrm{~g}), \quad \alpha-\mathrm{SM}$ actin $(r=+0.640, P<0.001)$ (Fig. 6 h), cell number $(r=+0.441$, $P<0.05)$ (Fig. 6i), GS score $(r=0.826, P<0.001)$ (Fig. 6j), and proteinuria $(r=+0.364, P<0.05)$ (Fig. 6k). However, neither AGT nor ang II showed a significant correlation with expression of ACE, AT1R, and hematuria index. Finally, a positive correlation was demonstrated between TGF- $\beta$ and $\alpha$-SM actin $(r=+0.625, P<0.001)$ (Fig. 61).
Effect of ang II on the AGT mRNA and protein levels by human GEC

The regulation of AGT expression by GEC and a possible role of GEC-AGT in disease progression of IgAN were examined using cultured human GEC. The addition of $10^{-7} \mathrm{M}$ ang II rapidly induced AGT mRNA expression of quiescent human GEC after stimulation for $1 \mathrm{~h}$. This increase peaked at $3 \mathrm{~h}$, with a sevenfold increase, and then gradually decreased during the course of the experiment (Fig. 7a). When the response of AGT transcript to ang II dose was investigated after incubation for $3 \mathrm{~h}$, a dosedependent increase between $10^{-11}$ and $10^{-7} \mathrm{M}$ was seen (Fig. 7b). Western blot analysis revealed a time-dependent increase of AGT protein product to $10^{-7} \mathrm{M}$ ang II stimulation. The increased expression of AGT protein followed the increase of AGT mRNA by GEC and peaked $\mathbf{a}$

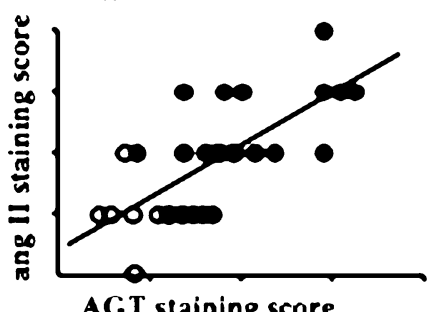

e

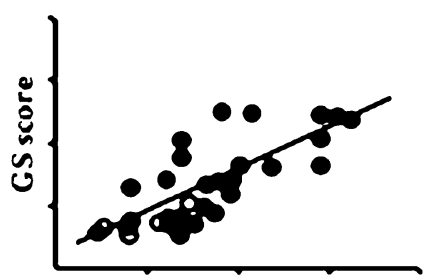

AGT staining score

i

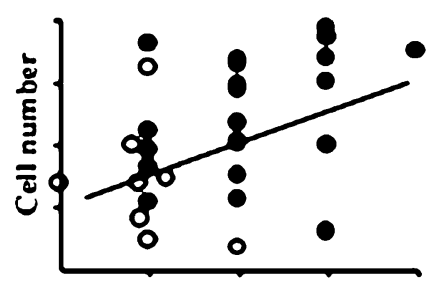

ang II staining score b

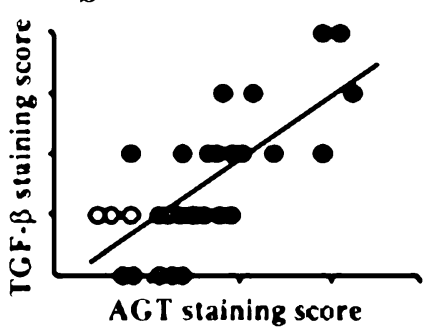

f

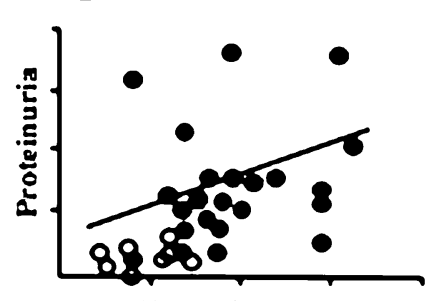

AGT staining score

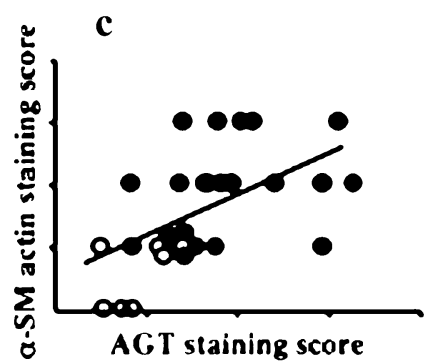

d

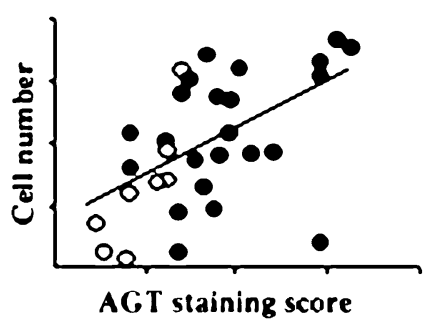

g
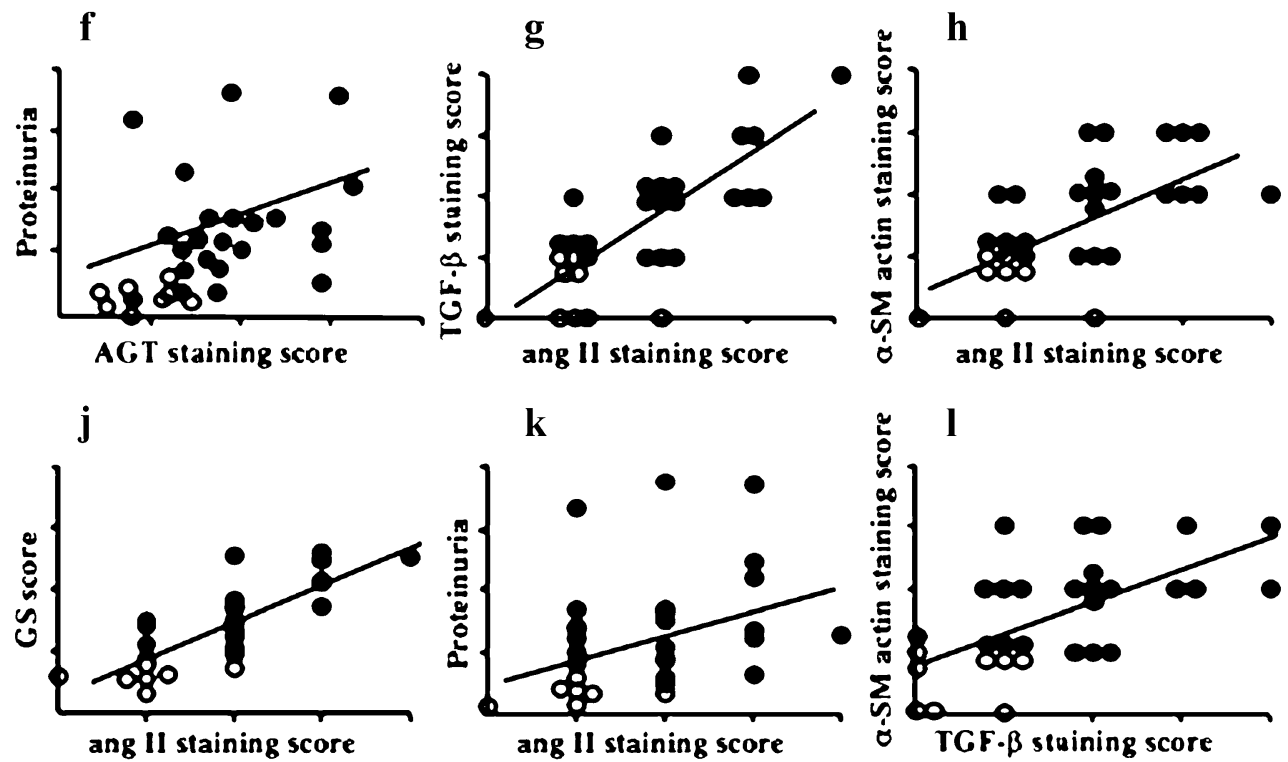

Fig. 6 Plots of correlations between the staining levels of angiotensinogen (AGT) and angiotensin II (ang II) (a), AGT and transforming growth factor- $\beta$ (TGF- $\beta$ ) (b), AGT and $\alpha$-smooth-muscle $(\alpha$-SM) actin (c), AGT and glomerular cell number (cell number) (d), AGT and glomerulosclerosis (GS) score (e), AGT and proteinuria (f), ang II and TGF- $\beta$ (g), ang II and $\alpha$-SM actin (h), ang II and cell number (i), ang II and GS score (j), ang II and proteinuria (k), and TGF- $\beta$ and $\alpha-S M$ actin (l) in patients with minor glomerular abnormalities (MGA) $(0)$ or immunoglobulin A nephropathy $(\operatorname{IgAN})(\bullet)$ (correlation; $P$ value) The

k

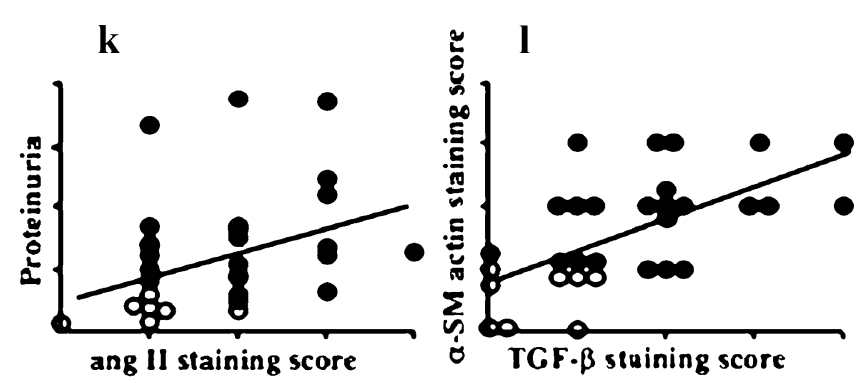

levels of AGT positively correlate with the level of ang II $(r=+0.711$; $P<0.001)$, TGF- $\beta(r=+0.723 ; P<0.001)$, cell number $(r=+0.542 ; P<$ $0.005)$, GS score $(r=+0.768 ; P<0.001)$, and proteinuria $(r=+0.436$; $P<0.05)$. Ang II is also positively correlated with TGF- $\beta(r=+0.782$; $P<0.001), \alpha$-SM actin $(r=+0.640 ; P<0.001)$, cell number $(r=+$ $0.441 ; P<0.05)$, GS score $(r=+0.826 ; P<0.001)$, and proteinuria $(r=$ $+0.364 ; P<0.05)$. The levels of TGF- $\beta$ positively correlate with the level of $\alpha$-SM actin $(r=+0.625 ; P<0.001)$ 
a

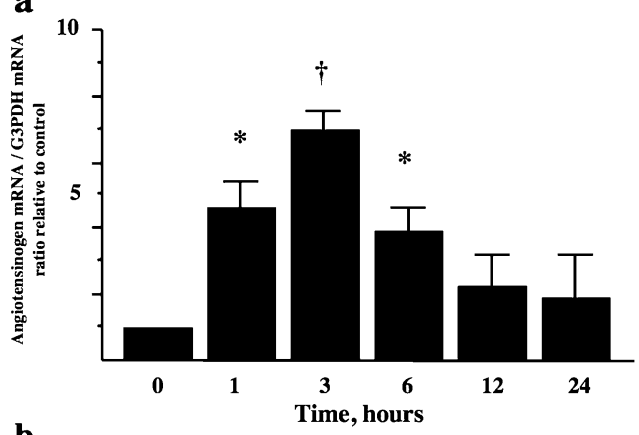

b

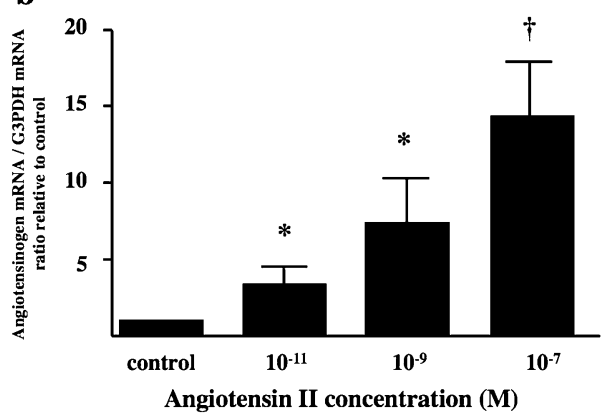

Fig. 7 Effects of angiotensin II (ang II) on the expression of angiotensinogen (AGT) messenger ribonucleic acid (mRNA) by cultured human glomerular endothelial cells (GEC). Human GEC were incubated with $10^{-7} \mathrm{M}$ ang II for the indicated time, extracted, and analyzed for AGT mRNA levels by real-time polymerase chain reaction (RT-PCR) (a). Values are means \pm standard deviation (SD). $* P<0.05$ and $\dagger P<0.01$ vs time 0 . Human GEC were incubated for 3 $\mathrm{h}$ with vehicle control or with indicated doses of ang II, extracted, and analyzed for AGT mRNA levels by RT-PCR (b). The graph represents the mean $\pm \mathrm{SD}$ of at least three independent experiments. ${ }^{*} P<0.05$ and $\uparrow P<0.01$ vs control

at 12 and $24 \mathrm{~h}$ after stimulation (Fig. 8a). Consistent with the results of RT-PCR analysis, a dose-dependent increase of human GEC-AGT protein to ang II stimulation was observed between $10^{-11} \mathrm{M}$ and $10^{-7} \mathrm{M}$ (Fig. 8b).

\section{Discussion}

Enhanced intrarenal RAS has been implicated in the development of hypertension and the progression of chronic renal diseases, including IgAN $[1,2,17]$. Ang II, the major effector molecule of the RAS cascade, is a key mediator of glomerular hypertension as well as glomerular sclerosis through the induction of TGF- $\beta[5,6]$. However, glomerular expression of RAS components and their association with local RAS activity and injury has not been extensively studied in human subjects, especially pediatric patients with IgAN.

In this study, we demonstrated for the first time that both glomerular expression of AGT protein, a sole substrate for RAS cascade, and ang II were strikingly increased with GEC and MC in nephritic glomeruli of pediatric IgAN. As it is already known that ang II is locally produced within the glomerulus and RAS is present in glomerular cell types such as GEC, MC, and podocyte [19, 20, 34], the increased expression of ang II seen in pediatric IgAN most likely reflects enhanced glomerular RAS activation under this disease setting.

We also demonstrated that there was a positive correlation between levels of glomerular AGT, ang II, and ang IIinducible factors (TGF- $\beta$ and $\alpha$-SM actin) and glomerular injury (cell proliferation and ECM accumulation), suggesting that the activated glomerular RAS cascade contributes to the development and progression of glomerular injury in pediatric IgAN. There are several studies investigating glomerular gene expression of RAS components in IgAN [17, 24, 35, 36]. Most studies have shown an increased expression of renin, $A G T$, and $A C E$ genes and decreased expression of $A T 1 R$ gene in nephritic glomeruli of IgAN. Furthermore, it has been shown that a negative feedback loop exists between ang II and its receptor, AT1R, in glomerular cells [36]. Therefore, if there was a proportional increase in ang II as a result of increased upstream components of RAS, a proportional

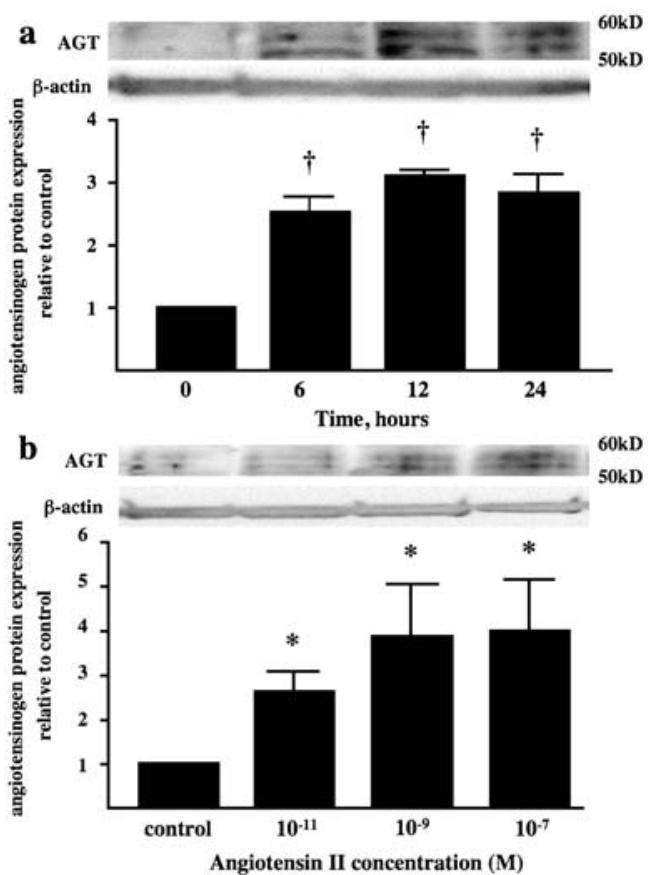

Fig. 8 Effects of angiotensin II (ang II) on the expression of angiotensinogen (AGT) protein by cultured human glomerular endothelial cells (GEC). Human GEC were incubated with $10^{-7} \mathrm{M}$ ang II for the indicated time, extracted, and then subjected to Western blot analysis for AGT and $\beta$-actin (a). Western blot analysis demonstrated two specific immunoreactive bands between 50 and $60 \mathrm{kD}$. Data for AGT protein levels were normalized by $\beta$-actin protein levels. The graph represents the mean \pm standard deviation (SD) of at least three independent experiments. $\dagger P<0.01$ vs time 0 . Human GEC were incubated for $12 \mathrm{~h}$ with vehicle control or with indicated doses of ang II, extracted, and analyzed, as described in a (b). Data are means $\pm \mathrm{SD}$ of at least three independent experiments. $* P<0.05$ vs control 
deactivation of $A T 1 R$ expression in IgAN would be expected. Here, we found a significantly greater expression of glomerular AGT protein and ang II peptides and a tendency for higher ACE protein levels and lower AT1R protein levels in pediatric IgAN compared with MGA patients, indicating that the glomerular AGT level may directly regulate the ang II activity under the coordination of ACE and AT1R expression and other ang II-producible enzyme activity. Various enzymes, including cathepsin and chymase, are involved in the RAS besides renin and ACE, which are classical enzyme synthesizing ang II from AGT [37]. Indeed, approximately $40 \%$ of the ang II found in the kidney is generated by ACE-independent pathways [38]. In any case, local levels of renin and ACE are not related to the synthesis of ang II in situations where AGT levels are very high [18, 20, 21]. Thus, the quantity of AGT might well be the most important element for the level of ang II production. Indeed, recent experimental studies using rat models of nephropathy indicated that enhanced intrarenal AGT expression is linked to the increased level of ang II production and contributes to the development of glomerular injury [20-22, 39].

We previously disclosed the mechanisms and the role of ang II-induced ECM deposition through MC [5, 6]. Ang II stimulates ECM synthesis through he induction of TGF- $\beta$ expression in cultured rat MC [5] and affects the plasminogen activator/plasminogen activator inhibitor-1 system to result in accelerated deposition of ECM [6], which indicates that ang II can act as a potent fibrotic molecule independent of its effects on blood pressure. Ang II can also induce MC activation ( $\alpha$-SM actin positive) [39-42]. In addition, experimental and human studies have shown that the degree of mesangial cellularity is associated with a high sensitivity to beneficial effects of ACEi and ARB on glomerular structure and proteinuria, suggesting that the glomerular ang II level may contribute to proliferation of MC [43]. Thus, our findings obtained here that the level of glomerular ang II is associated with the levels of TGF- $\beta$ and $\alpha$-SM actin expression as well as glomerular injury characterized by cell proliferation and ECM accumulation are consistent with the recent view of ang II action.

GEC damage and dysfunction are early features of many types of GN [44, 45]. Injury to GEC induces the secretion of various mediators, such as vasoactive substances, cytokines, and growth factors critical in the regulation of glomerular capillary tone and glomerular remodeling [19, 46-48]. Lee et al. demonstrated that GEC injury results in increased AGT and TGF- $\beta$ mRNA by GEC and MC in the subtotal nephrectomized rat model, suggesting that activated or injured endothelial cells synthesize AGT, triggering a cascade of ang II, TGF- $\beta$, and ECM protein gene expression with resultant development of the glomerular sclerotic lesion [19]. Here we provide in vivo data showing a striking increase of GEC-AGT and ang II in patients with
IgAN as well as in vitro data indicating the potential of GEC to synthesize AGT protein and stimulatory effects of ang II on GEC-AGT expression. Taken together, it seems likely that GEC plays a crucial role in the activation of local RAS, and self-amplifying properties of ang II production in GEC are associated with the glomerular hemodynamic changes and the development of progressive glomerular injury in human GN.

In general, RAS inhibitors are commonly used by chronic IgAN patients with hypertension or those with heavy proteinuria or initial impairment of renal function. We should stress that glomerular RAS activation could occur in pediatric IgAN without heavy proteinuria, hypertension, and renal impairment. IgAN patients in this study had a short-term observation period from onset to time of renal biopsy, renal lesions, characterized with mesangial proliferative changes, low-grade interstitial fibrosis, and tubular atrophy, indicating that they were diagnosed at an early stage of $\operatorname{IgAN}[14,49]$. Thus, considering the accumulating evidence that ang II plays a critical role in MC activation as well as glomerular hemodynamic changes and development of glomerulosclerosis, early treatment with $\mathrm{ACEi}$ and/or $\mathrm{ARB}$ in pediatric $\operatorname{IgAN}$, even in the absence of hypertension and/or severe proteinuria, is reasonable for preventing progression in $\operatorname{IgAN}$.

In conclusion, our study showed that activated glomerular AGT expression is likely to be involved in local RAS activation, leading to enhanced local ang II production, in pediatric IgAN with normal blood pressure and normal renal function and, thereby, may trigger a dangerous RAS cascade resulting in overproduction of TGF- $\beta$ and progression of glomerular injury. Moreover, a self-amplification loop of GEC-AGT production with ang II stimulation seems to drive the further injury of nephritic glomeruli in IgAN patients. These data provide a rationale for early intervention with RAS inhibitors in pediatric IgAN patients even in the absence of hypertension and/or impairment of renal function to prevent progression of glomerular injury.

Acknowledgments This work was supported by grants from the Japanese Ministry of Welfare $(16591035,18591189)$. We thank Dr. Hiroshi Kawachi and Dr. Fujio Shimizu (Niigata University, Niigata, Japan) for providing rabbit anti-TGF- $\beta$ antibody. We also thank Dr. Toshiaki Sano and Mrs. Naomi Okamoto for their excellent technical assistance. Parts of this work were published in abstract form at the 39th Annual Meeting of the American Society of Nephrology, 14-19 November 2006.

Open Access This article is distributed under the terms of the Creative Commons Attribution Noncommercial License which permits any noncommercial use, distribution, and reproduction in any medium, provided the original author(s) and source are credited. 


\section{References}

1. Anderson S, Rennke HG, Brenner BM (1986) Therapeutic advantage of converting enzyme inhibitors in arresting progressive renal disease associated with systemic hypertension in the rat. J Clin Invest 77:1993-2000

2. Navar LG, Harrison-Bernard LM, Imig JD, Wang CT, Cervenka L, Mitchell KD (1999) Intrarenal angiotensin II generation and renal effects of AT1 receptor blockade. J Am Soc Nephrol 10 (Suppl 12): S266-272

3. Nakamura N, Soubrier F, Menard J, Panthier JJ, Rougeon F, Corvol P (1985) Nonproportional changes in plasma renin concentration, renal renin content, and rat renin messenger RNA. Hypertension 7:855-859

4. Wagner J, Volk S, Haufe CC, Ciechanowicz A, Paul M, Ritz E (1995) Renin gene expression in human kidney biopsies from patients with glomerulonephritis or graft rejection. J Am Soc Nephrol 5:1469-1475

5. Kagami S, Border WA, Miller DE, Noble NA (1994) Angiotensin II stimulates extracellular matrix protein synthesis through induction of transforming growth factor- $\beta$ expression in rat glomerular mesangial cells. J Clin Invest 93:2431-2437

6. Kagami S, Kuhara T, Okada K, Kuroda Y, Border WA, Noble NA (1997) Dual effects of angiotensin II on the plasminogen/plasmin system in rat mesangial cells. Kidney Int 51:664-671

7. Ruiz-Ortega M, Egido J (1997) Angiotensin II modulates cell growth-related events and synthesis of matrix proteins in renal interstitial fibroblasts. Kidney Int 52:1497-1510

8. Mahmood J, Khan F, Okada S, Kumagai N, Morioka T, Oite T (2006) Local delivery of angiotensin receptor blocker into the kidney ameliorates progression of experimental glomerulonephritis. Kidney Int 70:1591-1598

9. Ruggenenti P, Perna A, Gherardi G, Gaspari F, Benini R, Remuzzi G (1998) Renal function and requirement for dialysis in chronic nephropathy patients on long-term ramipril: REIN follow-up trial. Gruppo Italiano di Studi Epidemiologici in Nefrologia (GISEN). Ramipril efficacy in nephropathy. Lancet 352:1252-1256

10. Horita Y, Tadokoro M, Taura K, Suyama N, Taguchi T, Miyazaki M, Kohno S (2004) Low-dose combination therapy with temocapril and losartan reduces proteinuria in normotensive patients with immunoglobulin a nephropathy. Hypertens Res 27:963-970

11. Ravid M, Brosh D, Levi Z, Bar-Dayan Y, Ravid D, Rachmani R (1998) Use of enalapril to attenuate decline in renal function in normotensive, normoalbuminuric patients with type 2 diabetes mellitus. A randomized, controlled trial. Ann Intern Med 128: 982-988

12. Coppo R, D, Amico G (2005) Factors predicting progression of IgA nephropathies. J Nephrol 18:503-512

13. Schena FP (1990) A retrospective analysis of the natural history of primary IgA nephropathy worldwide. Am J Med 89:209-215

14. Yoshikawa N, Iijima K, Maehara K, Yoshiara S, Yoshiya K, Matsuo T, Okada S (1987) Mesangial changes in IgA nephropathy in children. Kidney Int 32:585-589

15. Tanaka H, Suzuki K, Nakahata T, Tsugawa K, Konno Y, Tsuruga K, Ito E, Waga S (2004) Combined therapy of enalapril and losartan attenuates histologic progression in immunoglobulin A nephropathy. Pediatr Int 46:576-579

16. Yang Y, Ohta K, Shimizu M, Nakai A, Kasahara Y, Yachie A, Koizumi S (2005) Treatment with low-dose angiotensin-converting enzyme inhibitor (ACEI) plus angiotensin II receptor blocker (ARB) in pediatric patients with IgA nephropathy. Clin Nephrol 64:35-40

17. Del Prete D, Gambaro G, Lupo A, Anglani F, Brezzi B, Magistroni R, Graziotto R, Furci L, Modena F, Bernich P, Albertazzi A, D' Angelo A, Maschio G (2003) Precocious activation of genes of the renin-angiotensin system and the fibrogenic cascade in IgA glomerulonephritis. Kidney Int 64:149-159

18. Kobori H, Ozawa Y, Suzaki Y, Prieto-Carrasquro MC, Nishiyama A, Shoji T, Cohen EP, Navar LG (2006) Yong scholars award lecture: intratubular angiotensinogen in hypertension and kidney diseases. Am J Hypertens 19:541-550

19. Lee LK, Meyer TW, Pollock AS, Lovett DH (1995) Endothelial cell injury initiates glomerular sclerosis in the rat remnant kidney. J Clin Invest 96:953-964

20. Singh R, Singh AK, Leehey DJ (2005) A novel mechanism for angiotensin II formation in streptozotocin-diabetic rat glomeruli. Am J Physiol Renal Physiol 288:F1183-F1190

21. Kobori H, Ozawa Y, Suzaki Y, Nishiyama A (2005) Enhanced intrarenal angiotensinogen contributes to early renal injury in spontaneously hypertensive rats. J Am Soc Nephrol 16:2073-2080

22. Shin GT, Kim WH, Yim H, Kim MS, Kim H (2005) Effects of suppressing intrarenal angiotensinogen on renal transforming growth factor- $\beta 1$ expression in acute ureteral obstruction. Kidney Int 67:897-908

23. Churg J, Bernstein J, Glassock R (1995) Classification of glomerular disease. In: Churg J, Bernstein J, Glassoch R (eds), Renal disease: classification and atlas of glomerular disease, 2nd end. Igaku-Shoin, New York, pp 1-23

24. Miyake-Ogawa C, Miyazaki M, Abe K, Harada T, Ozono Y, Sakai H, Koji T, Kohno S (2005) Tissue-specific expression of renin-angiotensin system components in IgA nephropathy. Am J Nephrol 25:1-12

25. Kuhara T, Kagami S, Kuroda Y (1997) Expression of b1-integrins on activated mesangial cells in human glomerulonephritis. J Am Soc Nephrol 8:1679-1687

26. Ballardie FW, Roberts IS (2002) Controlled prospective trial of prednisolone and cytotoxics in progressive IgA nephropathy. J Am Soc Nephrol 13:142-148

27. Kondo S, Shimizu M, Urushihara M, Tsuchiya K, Yoshizumi M, Tamaki T, Nishiyama A, Kawachi H, Shimizu F, Quinn MT, Lambeth DJ, Kagami S (2006) Addition of the antioxidant probucol to angiotensin II type I receptor antagonist arrests progressive mesangioproliferative glomerulonephritis in the rat. J Am Soc Nephrol 17:783-794

28. Abe K, Miyazaki M, Koji T, Furusu A, Nakamura-Kurashige T, Nishino T, Ozono Y, Harada T, Sakai H, Kohno S (2001) Enhanced expression of complement C5a receptor mRNA in human diseased kidney assessed by in situ hybridization. Kidney Int 60:137-146

29. Suzaki Y, Ozawa Y, Kobori H (2006) Quantification of human angiotensinogen by a novel sandwich ELISA. Peptides 27:3000-3002

30. Murakami S, Morioka T, Nakagawa Y, Suzuki Y, Arakawa M, Oite T (2001) Expression of adhesion molecules by cultured human glomerular endothelial cells in response to cytokines: comparison to human umbilical vein and dermal microvascular endothelial cells. Microvasc Res 62:383-391

31. Gimenez-Roqueplo AP, Celerier J, Lucarelli G, Corvol P, Jeunemaitre X (1998) Role of N-glycosylation in human angiotensinogen. J Biol Chem 273:21232-21238

32. Wake DJ, Rask E, Livingstone DE, Soderberg S, Olsson T, Walker BR (2003) Local and systemic impact of transcriptional upregulation of 11b-hydroxysteroid dehydrogenase type 1 in adipose tissue in human obesity. J Clin Endorinol Metab 88:3983-3988

33. Kobori H, Harrison-Bernard LM, Navar LG (2001) Expression of angiotensinogen mRNA and protein in angiotensin II-dependent hypertension. J Am Soc Nephrol 12:431-439

34. Liebau MC, Lang D, Bohm J, Endlich N, Bek MJ, Witherden I, Mathieson PW, Saleem MA, Pavenstadt H, Fischer KG (2006) Functional expression of the renin-angiotensin system in human podocytes. Am J Physiol Renal Phyiol 290:710-719

35. Lai KN, Leung JC, Lai KB, To WY, Yeung VT, Lai FM (1998) Gene expression of the renin-angiotensin system in human kidney. J Hypertens 16:91-102 
36. Lai KN, Chan LY, Tang SC, Tsang AW, Li FF, Lam MF, Lui SL, Leung JC (2004) Mesangial expression of angiotensin II receptor in IgA nephropathy and its regulation by polymeric IgA1. Kidney Int 66:1403-1416

37. Brewster UC, Perazella MA (2004) The renin-angiotensinaldosterone system and the kidney: Effects on kidney disease. Am J Med 116:263-272

38. Hollenberg NK (1999) Pharmacologic interruption of the reninangiotensin system and the kidney: differential responses to angiotensin-converting enzyme and renin inhibition. J Am Soc Nephrol 10 (Suppl 11):S239-S242

39. Graciano ML, Cavaglieri Rde C, Delle H, Dominguez WV, Casarini DE, Malheiros DM, Noronha IL (2004) Intrarenal ReninAngiotensin system is upregulated in experimental model of progressive renal disease induced by chronic inhibition of nitric oxide synthesis. J Am Soc Nephrol 15:1805-1815

40. Johnson RJ, Iida H, Alpers CE, Majesky MW, Schwartz SM, Pritzi P, Gordon K, Gown AM (1991) Expression of smooth muscle cell phenotype by rat mesangial cells in immune complex nephritis. $\alpha$-smooth muscle actin is a marker of mesangial cell proliferation. J Clin Invest 87:847-858

41. Gomez-Garre D, Ruiz-Ortega M, Ortego M, Largo R, Lopez-Armada MJ, Plaza JJ, Gonzalez E, Egido J (1996) Effects and interactions of endothelin-1 and angiotensin II on matrix protein expression and synthesis and mesangial cell growth. Hypertension 27:885-892

42. Johnson RJ, Alpers CE, Yoshimura A, Lombardi D, Pritzl P, Floege J, Schwartz SM (1992) Renal injury from angiotensin IImediated hypertension. Hypertension 19:464-474
43. Minutolo R, Balletta MM, Catapano F, Chiodini P, Tirino G, Zamboli P, Fuiano G, Russo D, Marotta P, Iodice C, Conte G, De Nicola L (2006) Mesangial hypercellularity predicts antiproteinuric response to dual blockade of RAS in primary glomerulonephritis. Kidney Int 70:1170-1176

44. Hernandez E, Toledo T, Alamo C, Mon C, Rodicio JL, Praga M (1997) Elevation of von Willebrand factor levels in patients with IgA nephropathy: effect of ACE inhibition. Am J Kidney Dis 30:397-403

45. Kawasaki Y, Suzuki J, Nozawa R, Sakai N, Tannji M, Isome M, Suzuki H, Nozawa Y (2004) FB21, a monoclonal antibody that reacts with a sialic-acid-dependent carbohydrate epitope, is a marker for glomerular endothelial cell injury. Am J Kidney Dis 44:239-249

46. Ruiz-Ortega M, Ruperez M, Lorenzo O, Esteban V, Blanco J, Mezzano S, Egido J (2002) Angiotensin II regulates the synthesis of proinflammatory cytokines and chemokines in the kidney. Kidney Int Suppl 82:12-22

47. Wolf G, Ziyadeh FN, Zahner G, Stahl RA (1996) Angiotensin II is mitogenic for cultured rat glomerular endothelial cells. Hypertension 27:897-905

48. Cresci B, Giannini S, Pala L, Mavilia C, Manuelli C, Cappugi P, Maggi E, Rotella CM (2003) AT1 and AT2 receptors in human glomerular endothelial cells at different passages. Microvasc Res 66:22-29

49. Okada K, Funai M, Kawakami K, Kagami S, Yano I, Kuroda Y (1990) IgA nephropathy in Japanese children and adults: a comparative study of clinicopathological features. Am J Nephrol 10:191-197 